\title{
Circulating miRNAs and Cytokines Uncover Novel Physiopathological Mechanisms of COVID-19
}

\author{
Pedro Martínez-Fleta \\ Hospital Universitario de la Princesa
}

Paula Vera-Tomé

Hospital Universitario de la Princesa

María Jiménez-Fernández

Hospital Universitario de la Princesa

\section{Silvia Requena}

Hospital Universitario de la Princesa

\section{Emilia Roy-Vallejo}

Hospital Universitario de la Princesa

\section{Ancor Sanz-García}

Hospital Universitario de la Princesa

\section{Marta Lozano-Prieto}

Hospital Universitario de la Princesa

\section{Celia López-Sanz}

Hospital Universitario de la Princesa

\section{Alicia Vara}

Hospital Universitario de la Princesa

Ángel Lancho-Sánchez

Hospital Universitario de la Princesa

\section{Enrique Martín-Gayo}

Hospital Universitario de la Princesa

Cecilia Muñoz-Calleja

Hospital Universitario de la Princesa

\section{Arantzazu Alfranca}

Hospital Universitario de la Princesa

Isidoro González-Álvaro

Hospital Universitario de la Princesa José María Galván-Román

Hospital Universitario de la Princesa

Javier Aspa

Hospital Universitario de la Princesa

Hortensia de la Fuente

Hospital Universitario de la Princesa 
Francisco Sanchez-Madrid ( $\square$ fsmadrid@salud.madrid.org )

Hospital Universitario de la Princesa https://orcid.org/0000-0001-5303-0762

\section{Research Article}

Keywords: COVID-19, community-acquired pneumonia, microRNAs, soluble proteins

Posted Date: November 2nd, 2021

DOl: https://doi.org/10.21203/rs.3.rs-976578/v1

License: (c) (i) This work is licensed under a Creative Commons Attribution 4.0 International License. Read Full License 


\section{Abstract}

Coronavirus Disease 2019 (COVID-19) pneumonia is a life-threatening infectious disease, especially for elderly patients with multiple comorbidities. Despite enormous efforts to understand its underlying etiopathogenic mechanisms, most of them remain elusive. In this study, we compared differential plasma miRNAs and cytokines profiles between COVID-19 and other community-acquired pneumonias (CAP). A first screening and subsequent validation assays in an independent cohort of patients revealed a signature of 15 dysregulated miRNAs between COVID-19 and CAP patients. Additionally, multivariate analysis displayed a combination of 4 miRNAs (miR-106b-5p, miR-221-3p, miR-25-3p and miR-30a-5p) that significantly discriminated between both pathologies. Search for targets of these miRNAs, combined with plasma protein measurements, identified a differential cytokine signature between COVID-19 and CAP that included EGFR, CXCL12 and IL-10. Significant differences were also detected in plasma levels of CXCL12, IL-17, TIMP-2 and IL-21R between mild and severe COVID-19 patients. These findings provide new insights into the etiopathological mechanisms underlying COVID-19.

\section{Introduction}

Severe Acute Respiratory Syndrome Coronavirus 2 (SARS-CoV-2) is responsible for the disease known as coronavirus disease 2019 (COVID-19) [1]. Although initially COVID-19 was defined as a low tract respiratory disease, it is now acknowledged as a complex disorder that compromises multiple organs and may cause long-lasting damage even in patients that overcome the acute phase [2]. Major efforts have been devoted to identifying molecules able to predict the severity of COVID-19. These efforts were aimed to improve patient stratification and make a better use of the available resources to optimise health care. After one year of intensive research, some prognostic clinical biomarkers have been identified, including lymphocyte count, Ddimer, C reactive protein (CRP), ferritin, Interleukin (IL)-6 or viremia [3, 4]. Moreover, dysregulation of other cytokines, including IL-2, IL-10, Interferon (IFN)- $y$, monocyte chemoattractant protein (MCP)-1 or C-X-C Motif Chemokine Ligand (CXCL) 10 in COVID-19 patients have also been reported [5].

MicroRNAs (miRNAs) are short (22 nucleotides in length on average) non-coding RNAs that function as posttranscriptional regulators by binding to mRNAs and preventing protein translation. Each miRNA can target multiple genes, which makes them important regulators of numerous cellular functions [6]. Furthermore, miRNAs mediate cell to cell communication travelling either as free circulating molecules or inside exosomes, thereby modulating multiple immune system functions or inflammation in several diseases [7-9]. These molecules can be used to predict the clinical course of patients with viral infections, since certain miRNAs are able to bind to distinct RNA viral genomes, blocking their replication [10]. A specific miRNA signature has been determined for other viruses, e.g. influenza, which led researchers to find dysregulated pathways in critically ill patients [11].

In silico analyses of potentially relevant miRNAs in COVID-19 have been previously carried out. These studies focused on identifying host miRNAs specifically targeting the SARS-CoV-2 genome as a defence strategy [12] or viral miRNAs affecting expression of host genes that could be associated with the pathogenesis of the disease [13]. However, to our knowledge, no studies have been published that identify miRNAs specifically dysregulated by SARS-CoV-2 infection compared to other types of pneumonia. 
The aim of this work was to determine a profile of plasma miRNAs and soluble target molecules in COVID-19 compared to community-acquired pneumonia (CAP) as a specificity control group. CAP was selected because it comprises a group of low tract respiratory infections whose symptoms frequently resemble those of SARS-CoV-2 infection. Here, we identify circulating molecules, such as miRNAs and cytokines, which are differentially expressed in COVID-19 compared to CAP. This study provides novel insights into the molecular mechanisms of this pathology.

\section{Materials And Methods}

\section{Experimental design and patient selection}

To ensure an unbiased manipulation of the samples, a blinded study was carried out. A total of 123 COVID19 patients were included in this retrospective study. They all had been diagnosed by a positive result of RTqPCR for SARS-CoV-2 in nasopharyngeal swabs. Patients were admitted to the University Hospital de La Princesa from March 10th to April 21st, 2020 (first wave). Plasma samples were collected within 5 days upon admission. To better characterise COVID-19 patients, serological tests to detect IgG, IgA, and IgM antibodies against the SARS-CoV-2 spike (S) and the nucleocapsid viral protein (NP) were performed (Supplementary Fig. 1).

COVID-19 patients were mainly treated with viral protease inhibitors (lopinavir/ritonavir), hydroxychloroquine and/or azithromycin, according to local therapeutic guidelines at the time. Patients treated with either corticosteroids or tocilizumab were excluded from the study. Demographic and clinical variables of the study populations were collected.

The CAP cohort consisted of 33 adult patients, presenting symptoms of lower respiratory tract infection together with the appearance of a new infiltrate on a chest radiograph and the absence of an alternative diagnosis during follow-up, according to the usual definition. All of them were diagnosed with CAP and admitted to the University Hospital de La Princesa. This cohort has been previously used in other studies, in the context of research project on prognostic biomarkers in CAP $[14,15]$. Plasma samples were collected at admission, according with ATS/IDSA guidelines in force [16]. These samples were obtained from the Biobank of the University Hospital de La Princesa and they have been included before pandemic.

Our screening cohort was composed of 38 COVID-19 patients, 20 with mild disease and 18 with severe disease, classified according to symptoms severity [17]. The validation cohort comprised 43 mild and 42 severe COVID-19 patients (Supplementary Fig. 2).

\section{Human plasma extraction and RNA purification from COVID- 19 patients}

To collect the plasma, EDTA tubes with $10 \mathrm{ml}$ of venous peripheral blood were centrifuged $20 \mathrm{~min}$ at $2000 \mathrm{xg}$ at $4^{\circ} \mathrm{C} .1 \mathrm{ml}$ of plasma was then aliquoted and stored at $-80^{\circ} \mathrm{C}$ for RNA extraction.

RNA was isolated from $200 \mu \mathrm{l}$ of plasma using miRNeasy Serum/Plasma Advanced Kit (QIAGEN), following the manufacturer's instructions. Plasma samples were centrifuged 5 minutes at $4^{\circ} \mathrm{C}$ and $3000 \times \mathrm{g} .1 .25$ 
$\mu \mathrm{g} / \mathrm{ml}$ MS2 bacteriophage RNA (Roche Diagnostics) and $1 \mu \mathrm{l}$ of UniSp2, UniSp4 and UniSp5 RNA spike-in templates were added to each sample as a quality control of the RNA extraction process. The RNA was eluted in $20 \mu \mathrm{l}$ of RNase-Free Water and then stored at $-80^{\circ} \mathrm{C}$ until use.

To assess hemolysis, absorbance (Abs) at $414 \mathrm{~nm}$ of all plasma samples was measured using NanoDrop One/One ${ }^{\mathrm{C}}$ Spectrophotometer (Thermofisher Scientific) and those severely hemolysed samples (Abs > 5) were discarded.

\section{Reverse transcription (RT) and RT-qPCR assays}

The isolated RNA was reverse transcribed to complementary DNA (CDNA) using miRCURY LNA RT Kit (QIAGEN) following manufacturer's instructions and quality control of this process was carried out using celmiR-39 and Unisp6 RNA spike-in templates.

The screening of the candidate miRNAs by qPCR was performed by means of miRCURY LNA miRNA Focus PCR Panels (QIAGEN), which comprises 179 commonly found miRNAs in human plasma.

For qPCR validation assays, miRCURY LNA miRNA Custom PCR Panels (QIAGEN) were used. Both assays were performed using miRCURY LNA SYBR® Green PCR Kit (QIAGEN). QuantStudio 5 Real-Time PCR System (ThermoFisher Scientific) was used for qPCR plates reading.

\section{qPCR data analysis}

A quality control of the RNA extraction was performed by means of the spike-in previously added to the extraction and outlier samples were removed from the analysis.

Ct values above 36 were discarded for the global mean calculation. Interplate calibration (IPC) was performed using UniSp3 as described in the kit's Handbook. Then, Relative Quantities (RQ) were calculated as the log base 2 values of the Ct difference between miRNAs and UniSp2 RNA spike-in as described elsewhere [18]. Normalisation factor (NF) was calculated as the geometric mean of the RQs of all expressed targets per sample. Normalised Relative Quantities (NRQ) were obtained by dividing the RQs by the sample specific NF.

Data from discovery cohort were normalised using the Global Mean normalisation method, as previously described [19]. For the miRNAs validation experiments, a group of stable miRNAs was selected from the screening panel using the software packages Normfinder and geNorm. miR-15b-5p, miR-30d-5p, let-7i-5p and miR-15a-5p were chosen to normalise data of the COVID-19 mild vs. severe analysis. Normalisation of CAP and COVID-19 analysis was performed using miR-103a-3p, miR-320a, miR-30e-5p and miR-15b-5p.

\section{Target validation by ELISA arrays}

Plasma levels of soluble cytokines and proteins were analysed using a custom ELISA multiplex array (RayBiotech Life, GA, USA) according to manufacturer's instructions.

\section{Expression of the SARS-CoV-2 nucleocapsid and Spike proteins}


Nucleocapsid (NP) protein construct was expressed in the E. coli strain BL21 Star (DE3) pLysS (ThermoFisher) and purified as described before [20].

Recombinant cDNA coding for soluble S (residues 1 to 1208) protein was cloned in the pcDNA3.1 vector for expression in HEK-293F cells using standard transfection methods. The construct contained the $S$ signal sequence at the $\mathrm{N}$-terminus, and a T4 fibritin trimerization sequence, a Flag epitope, and an 8xHis-tag at the C-terminus. The furin-recognition motif (RRAR) was replaced by the GSAS sequence, and it contained the A942P, K986P and V987P substitutions in the S2 portion. Proteins were purified by Ni-NTA affinity chromatography from transfected cell supernatants, and they were transferred to $25 \mathrm{mM}$ Hepes-buffer and $150 \mathrm{mM} \mathrm{NaCl}, \mathrm{pH}$ 7.5, during concentration.

\section{ELISA for detection of antibodies to SARS-CoV-2}

96-well Maxisorp Nunc-Immuno plates were coated with $100 \mu \mathrm{l} /$ well of recombinant proteins diluted in borate buffered saline (BBS); NP at $0.5 \mu \mathrm{g} / \mathrm{ml}$ and $\mathrm{S}$ at $1 \mu \mathrm{g} / \mathrm{ml}$ and incubated overnight at $4^{\circ} \mathrm{C}$. Coating solutions were then aspirated, ELISA plates were washed three times with $200 \mu \mathrm{l}$ of PBS $0.05 \%$ Tween 20 (PBS-T) and then dried before blocking with PBS-casein (Biorad, 1x PBS blocker) for $1 \mathrm{~h}$ at room temperature. The plates were washed again with PBS-T and $100 \mu$ of patient serum/plasma sample diluted in PBS-casein, $0.02 \%$ Tween-20, as indicated, were added, and incubated for $2 \mathrm{~h}$ at room temperature. The plates were washed again and $100 \mu \mathrm{l} /$ well of the indicated detection antibody (AffiniPure Rabbit Anti-Human IgM, Fc $\mu$ fragment specific. HRPO, AffiniPure Rabbit Anti-Human Serum IgA, a chain specific. HRPO, or AffiniPure Rabbit AntiHuman IgG, Fcy fragment specific. HRPO, all from Jackson Labs) were added and incubated for $1 \mathrm{~h}$ at room temperature. The plates were washed with PBS-T four times and incubated at room temperature in the dark with $100 \mu \mathrm{l} /$ well of Substrate Solution (OPD, Sigma prepared according to the manufacturer's instructions) (typically for $3 \mathrm{~min}$ ), $50 \mu \mathrm{l}$ of stop solution (3M H2SO4) were then added to each well and the optical density (at $492 \mathrm{~nm}$ ) of each well was determined using a microplate reader.

\section{Statistical analysis}

Categorical variables were represented by absolute value and percentage, and continuous variables were represented by median and interquartile range (IQR).

A one-way ANOVA was used to select potential candidate miRNAs with significant differences $(p<0.05)$ across groups (CAP and mild and severe COVID-19 patients) in the screening analysis. Then, of those potential candidates, a multiple linear regression analysis for miRNAs following normal distribution or a logistic regression analysis for miRNAs which could not be transformed to normally-distributed variables were used. Quantitative variables following a non-normal distribution were transformed to normally distributed variables. These models were adjusted by confounding variables such as sex, age, days postonset of symptoms (POS), ethnic group, or hemolysis. To search for possible confounding variables, Pearson or Spearman correlations were employed. False discovery rate (FDR) correction was assessed by the Benjamini-Hochberg method [21].

Logistic regression analysis for CAP and COVID-19 classification was characterised by using all the variables with a stepwise procedure, with both backward and forward search based on Akaike information criteria, to 
select the critical variables. The discrimination validity of the score, and also the main value, were assessed by AUC of the ROC curve along with the $95 \%$ confidence interval $(95 \% \mathrm{Cl})$.

A Youden's $J$ statistic was used to determine the sensitivity and specificity of the model. These statistical analyses were performed using our own codes and base functions in R, version 3.5.1 (http://www.Rproject.org).

Graphs were performed with GraphPad Prism 8. For outlier detection and removal, ROUT algorithm with an FDR of $1 \%$ was used. The rest of the analyses were performed with Stata 14.0 for Windows (Stata Corp LP, College Station, TX).

\section{Results}

\section{Cohort selection and clinical features of study populations}

miRNA profile was assessed in a discovery cohort that consisted of 38 COVID-19 patients (20 with mild and 18 with severe disease), collected during the first wave of the pandemic in Spain, from March to April 2020. Median age was 59.5, 15 were female and 23 were male. High blood pressure (HBP) and dyslipidemia (DL) were the most common comorbidities found in the COVID-19 cohort (34.2\% and $29 \%$ respectively). As a specificity control group, 9 patients ( 5 female and 4 male) presenting with CAP were included, with a median age of 62.

The validation cohort comprised 85 patients with COVID-19 (43 with mild and 42 with severe disease), recruited during the first wave, and 24 CAP individuals. CAP patients were recruited before pandemic (20142015) and most of them were of unknown etiology. Median age was similar between both cohorts ( 66.5 and $64)$, whereas the CAP cohort had higher proportion of males (58.3\% versus $41.1 \%$ in COVID-19). Among comorbidities, DL (45.9\%) and cardiovascular disease (CD) (10.6\%) were most frequently found in COVID-19 individuals when compared with CAP ( $25 \%$ and $8.3 \%$ respectively). On the other hand, chronic obstructive pulmonary disease (COPD) was higher in CAP patients (20.8\% vs $9.4 \%)$. The main demographic and clinical characteristics of the study population are described in Table 1. 
Table 1

Demographic and clinical characteristics of the study population.

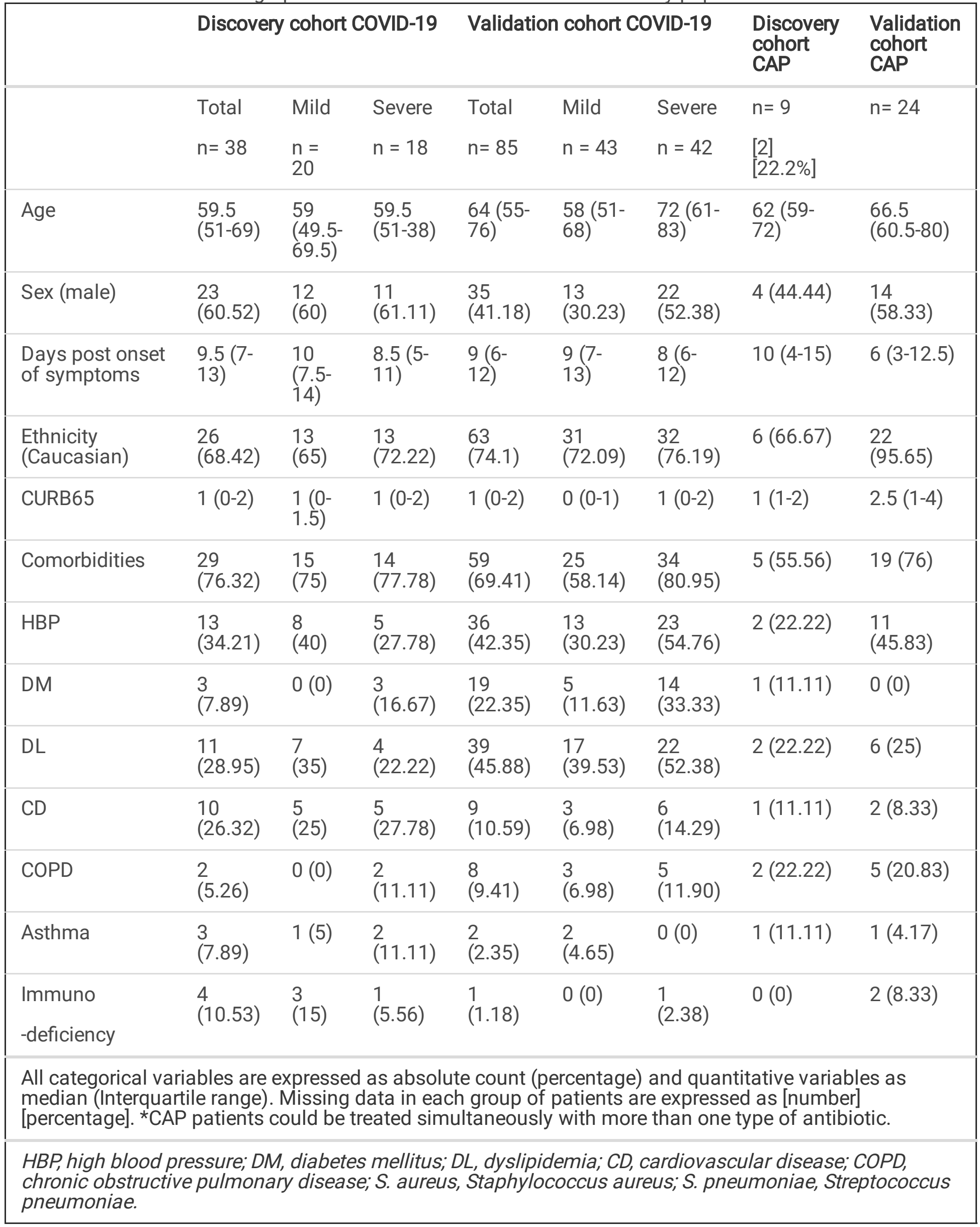




\begin{tabular}{|c|c|c|c|c|c|c|c|c|}
\hline \multirow[b]{2}{*}{ Acute treatment } & \multicolumn{3}{|c|}{ Discovery cohort COVID-19 } & \multicolumn{3}{|c|}{ Validation cohort COVID-19 } & \multirow{2}{*}{$\begin{array}{l}\begin{array}{l}\text { Discovery } \\
\text { cohort } \\
\text { CAP }\end{array} \\
7(77.78) \\
\end{array}$} & \multirow{2}{*}{$\begin{array}{l}\begin{array}{l}\text { Validation } \\
\text { cohort } \\
\text { CAP }\end{array} \\
24(100)\end{array}$} \\
\hline & $\begin{array}{l}38 \\
(100)\end{array}$ & $\begin{array}{l}20 \\
(100)\end{array}$ & $\begin{array}{l}18 \\
(100)\end{array}$ & $\begin{array}{l}82 \\
(96.47)\end{array}$ & $\begin{array}{l}40 \\
(93.02)\end{array}$ & $\begin{array}{l}42 \\
(100)\end{array}$ & & \\
\hline $\begin{array}{l}\text { Lopinavir/ } \\
\text { ritonavir }\end{array}$ & $\begin{array}{l}25 \\
(65.78)\end{array}$ & $\begin{array}{l}11 \\
(55)\end{array}$ & $\begin{array}{l}14 \\
(82.35)\end{array}$ & $\begin{array}{l}52 \\
(61.18)\end{array}$ & $\begin{array}{l}28 \\
(65.12)\end{array}$ & $\begin{array}{l}24 \\
(57.14)\end{array}$ & - & - \\
\hline $\begin{array}{l}\text { Hydroxy- } \\
\text { chloroquine }\end{array}$ & $\begin{array}{l}33 \\
(86.84)\end{array}$ & $\begin{array}{l}18 \\
(90)\end{array}$ & $\begin{array}{l}15 \\
(88.24)\end{array}$ & $\begin{array}{l}81 \\
(95.29)\end{array}$ & $\begin{array}{l}40 \\
(93.02)\end{array}$ & $\begin{array}{l}41 \\
(97.62)\end{array}$ & - & - \\
\hline Antibiotics* & $\begin{array}{l}26 \\
(68.42)\end{array}$ & $\begin{array}{l}15 \\
(75)\end{array}$ & $\begin{array}{l}11 \\
(64.71)\end{array}$ & $\begin{array}{l}69 \\
(81.17)\end{array}$ & $\begin{array}{l}37 \\
(86.05)\end{array}$ & $\begin{array}{l}32 \\
(76.19)\end{array}$ & $7(77.78)$ & $24(100)$ \\
\hline Beta lactam & - & - & - & - & - & - & $3(33.33)$ & $15(62.5)$ \\
\hline Quinolone & - & - & - & - & - & - & $4(44.44)$ & $3(12.5)$ \\
\hline $\begin{array}{l}\text { Macrolide } \\
\text { (Clarithromycin/ } \\
\text { Azithromycin) }\end{array}$ & $\begin{array}{l}26 \\
(68.42)\end{array}$ & $\begin{array}{l}15 \\
(75)\end{array}$ & $\begin{array}{l}11 \\
(64.71)\end{array}$ & $\begin{array}{l}69 \\
(81.17)\end{array}$ & $\begin{array}{l}37 \\
(86.05)\end{array}$ & $\begin{array}{l}32 \\
(76.19)\end{array}$ & $2(22.22)$ & $\begin{array}{l}20 \\
(83.33)\end{array}$ \\
\hline \multicolumn{9}{|l|}{$\begin{array}{l}\text { Isolated } \\
\text { pathogen }\end{array}$} \\
\hline SARS-CoV-2 & $\begin{array}{l}38 \\
(100)\end{array}$ & $\begin{array}{l}20 \\
(100)\end{array}$ & $\begin{array}{l}18 \\
(100)\end{array}$ & $\begin{array}{l}85 \\
(100)\end{array}$ & $\begin{array}{l}43 \\
(100)\end{array}$ & $\begin{array}{l}42 \\
(100)\end{array}$ & - & - \\
\hline Unknown & - & - & - & - & - & - & 7 (77.78) & $20(80)$ \\
\hline S. aureus & - & - & - & - & - & - & $1(11.11)$ & $1(4)$ \\
\hline S. pneumoniae & - & - & - & - & - & - & $1(11.11)$ & $3(12)$ \\
\hline \multicolumn{9}{|c|}{$\begin{array}{l}\text { All categorical variables are expressed as absolute count (percentage) and quantitative variables as } \\
\text { median (Interquartile range). Missing data in each group of patients are expressed as [number] } \\
\text { [percentage]. *CAP patients could be treated simultaneously with more than one type of antibiotic. }\end{array}$} \\
\hline $\begin{array}{l}\text { HBP, high blood } \\
\text { chronic obstruct } \\
\text { pneumoniae. }\end{array}$ & ulmo & abc & $\begin{array}{l}\text { nellitu } \\
\text { S. aure }\end{array}$ & $\begin{array}{l}d y s \\
\text { taph }\end{array}$ & $\begin{array}{l}\operatorname{mia} ; \\
\text { ccus }\end{array}$ & $\begin{array}{l}\text { rdiov } \\
\text { IS;S. }\end{array}$ & $\begin{array}{l}\text { ar diseas } \\
\text { moniae, }\end{array}$ & $\begin{array}{l}\text { COPD, } \\
\text { ptococcus }\end{array}$ \\
\hline
\end{tabular}

\section{Differentially expressed miRNAs between COVID-19 and CAP}

A screening of 179 miRNAs commonly found in plasma revealed the existence of great differences in the miRNA profile expression between COVID-19 and CAP patients. More than 40 miRNAs showed a significant differential expression (Figure 1a). Multiple linear regression analyses were then performed, adjusting by possible confounding variables such as sex, age, hemolysis or POS. Using this approach, we found 35 miRNAs expressed at significantly different levels with a FDR corrected $p$ value $<0.05$ (Figure $1 \mathrm{~b}$ and Table 2). Data analysis revealed 19 upregulated and 16 downregulated miRNAs in COVID-19 vs. CAP patients. To confirm these findings, 21 candidate miRNAs were selected for further validation in an extended cohort of 
patients (Supplementary Fig. 2). Candidate miRNAs were chosen based on their expression levels in plasma and their potential biological interest. To confirm the differences detected in the discovery cohort, individual RT-qPCR for every candidate miRNA was carried out in the validation cohort. Multivariate analyses adjusted by POS, age, sex, hemolysis or ethnic group was performed to probe statistical differences between miRNA levels in COVID-19 and CAP. A total of 15 miRNAs displayed differences between groups with a FDR corrected $\mathrm{p}$ value $<0.05$, as shown in Figure $1 \mathrm{c}$ and Supplementary Table 1. 
Table 2

Differentially expressed miRNAs in COVID-19 vs. CAP patients found in discovery cohort.

\begin{tabular}{|c|c|c|c|c|}
\hline \multirow{2}{*}{$\begin{array}{l}\text { miRNA } \\
\text { hsa-miR-140-3p }\end{array}$} & \multirow{2}{*}{$\begin{array}{l}\text { FDR corrected p-value }{ }^{e} \\
0.01790\end{array}$} & \multicolumn{2}{|c|}{ Confidence Interval 95\% } & \multirow{2}{*}{$\begin{array}{l}\text { COVID-19/CAP } \\
\uparrow\end{array}$} \\
\hline & & 0.5072 & 1.2578 & \\
\hline hsa-miR-335-5p & 0.00895 & -0.4236 & -0.1462 & $\downarrow$ \\
\hline hsa-miR-27b-3pa & 0.00597 & -1.1172 & -0.3485 & $\downarrow$ \\
\hline hsa-miR-660-5pa & 0.00448 & 0.3304 & 1.0587 & $\uparrow$ \\
\hline hsa-miR-130a-3pa & 0.00358 & -1.1136 & -0.505 & $\downarrow$ \\
\hline hsa-miR-16-5pa & 0.00298 & 0.5155 & 1.1372 & $\uparrow$ \\
\hline hsa-miR-146a-5pa & 0.00256 & -1.7077 & -0.7462 & $\downarrow$ \\
\hline hsa-miR-362-3p ${ }^{b}$ & 0.00224 & 0.0898 & 0.2843 & $\uparrow$ \\
\hline hsa-miR-425-5p & 0.00199 & 0.2963 & 0.6154 & $\uparrow$ \\
\hline hsa-miR-101-3p ${ }^{a}$ & 0.00179 & 0.33 & 0.939 & $\uparrow$ \\
\hline hsa-miR-376a-3p b & 0.00163 & -0.4307 & -0.1448 & $\downarrow$ \\
\hline hsa-miR-126-5pa & 0.00149 & -1.2407 & -0.5048 & $\downarrow$ \\
\hline hsa-miR-382-5p $p^{b}$ & 0.00138 & -0.2923 & -0.1191 & $\downarrow$ \\
\hline hsa-miR-451ab & 0.00128 & 7.8489 & 14.9547 & $\uparrow$ \\
\hline hsa-miR-19a-3pa & 0.00119 & 0.2923 & 0.8424 & $\uparrow$ \\
\hline hsa-miR-19b-3pa & 0.00112 & 0.2318 & 0.7337 & $\uparrow$ \\
\hline hsa-miR-24-3p ${ }^{b}$ & 0.00105 & -1.3138 & -0.612 & $\downarrow$ \\
\hline hsa-miR-199a-3pa & 0.00099 & - & - & $\downarrow$ \\
\hline hsa-miR-584-5p & 0.00942 & -0.3005 & 0.0837 & $\downarrow$ \\
\hline hsa-miR-16-2-3p & 0.00895 & 0.1146 & 0.3954 & $\uparrow$ \\
\hline hsa-miR-532-3p & 0.01705 & 0.0476 & 0.1899 & $\uparrow$ \\
\hline
\end{tabular}

All miRNAs passing FDR correction ( $p$-value<q-value) are shown. Multiple linear regression analyses were performed except for ${ }^{c}$ (logistic regression) and ${ }^{d}$ (Mann-Whitney tests). The arrows represent up or downregulation for each miRNA in COVID-19 with respect to CAP. miRNAs in bold were selected for validation assays. ${ }^{a}$ log transformed miRNAs, ${ }^{b}$ square root transformed miRNAs, ${ }^{c}$ variable categorisation. ${ }^{\mathrm{e}} \mathrm{q}$-value threshold: 0.1 . 


\begin{tabular}{|c|c|c|c|c|}
\hline \multirow{2}{*}{$\begin{array}{l}\text { miRNA } \\
\text { hsa-miR-424-5pa }\end{array}$} & \multirow{2}{*}{$\begin{array}{l}\text { FDR corrected p-value } \\
0.01627\end{array}$} & \multicolumn{2}{|c|}{ Confidence Interval 95\% } & \multirow{2}{*}{$\begin{array}{l}\text { COVID-19/CAP } \\
\uparrow\end{array}$} \\
\hline & & 0.2387 & 1.0115 & \\
\hline hsa-miR-25-3p & 0.01557 & 1.4206 & 6.1161 & $\uparrow$ \\
\hline hsa-miR-99b-5p b & 0.01492 & -0.2093 & -0.5208 & $\downarrow$ \\
\hline hsa-miR-361-5p & 0.02065 & -0.3181 & -0.0672 & $\downarrow$ \\
\hline hsa-miR-221-3pc & 0.01989 & -1.1492 & 0.8271 & $\downarrow$ \\
\hline hsa-miR-194-5p b & 0.03703 & 0.0689 & 0.3965 & $\uparrow$ \\
\hline hsa-miR-29c-3p & 0.04042 & 0.5399 & 3.2983 & $\uparrow$ \\
\hline hsa-miR-33a-5p b & 0.05034 & -0.375 & -0.0576 & $\downarrow$ \\
\hline hsa-miR-106b-5pa & 0.05424 & -3.4736 & -0.2214 & $\uparrow$ \\
\hline hsa-miR-126-3pa & 0.06137 & -1.2407 & -0.5048 & $\downarrow$ \\
\hline hsa-miR-502-3p & 0.06289 & 0.0187 & 0.148 & $\uparrow$ \\
\hline hsa-miR-186-5p & 0.07344 & 0.0166 & 0.1513 & $\uparrow$ \\
\hline hsa-miR-130b-3pc & 0.08055 & -4.882 & -0.4597 & $\downarrow$ \\
\hline hsa-miR-30a-5pc & 0.08098 & 0.4416 & 4.8825 & $\uparrow$ \\
\hline \multicolumn{5}{|c|}{ 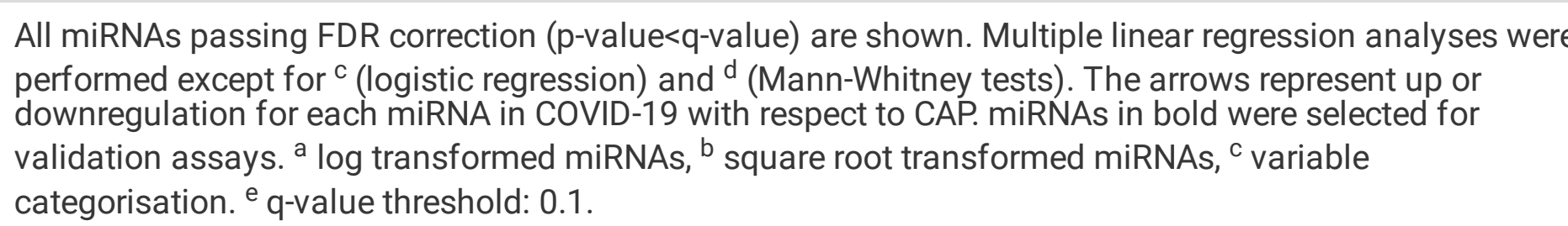 } \\
\hline
\end{tabular}

We next interrogated the possibility that mild and severe COVID-19 patients also displayed specific miRNA signatures. Due to subtle differences found in miRNAs expression between these groups, we chose those miRNAs with an absolute fold change in expression (Ct) higher than 1.3 and a raw $p$ value below 0.1 for further validation (Supplementary Table 2). Those miRNAs with a high number of non-detectable values in expression (above $50 \%$ of the samples) were not considered. Thereby, we found 10 potential candidate miRNAs (Supplementary Fig. 3). Only miR-185-5p was downregulated in severe cases of COVID-19. On the other hand, no statistically significant differences in the expression of the candidate miRNAs between COVID19 mild and severe patients were observed in the validation cohort (Supplementary Table 3).

\section{Logistic regression model based on miRNAs for classification of COVID-19/CAP patients}


Next, we developed a multivariate regression model able to differentiate and classify individuals with COVID19 and CAP. For that purpose, the miRNAs selected for validation assays and other variables including age, sex, ethnic group, POS, IL-6, IgG, IgA, IgM, C3 and C4 were considered. Additional clinical variables shown in Supplementary Table 4 and main comorbidities: HBP, diabetes mellitus (DM), DL, CD, COPD, asthma and secondary immunodeficiency were also included. Due to the high number of variables tested, a stepwise logistic regression procedure was employed that included the data corresponding to both discovery and validation cohorts. Among all these variables, we only found 4 miRNAs significantly contributing to the regression model: miR-106b-5p, miR-221-3p, miR-25-3p and miR-30a-5p. Hemolysis was included as confounding variable, showing no significant effect on the final model $(p=0.12)$. To assess the performance of this model, a Receiver Operating Characteristic (ROC) analysis was carried out, which displayed a high area under the curve (AUC) value: 0.952 (0.895-1), with a sensitivity of $93.75 \%$ and a specificity of $89.09 \%$ (Figure 2a).

\section{Analysis of pathways and miRNA targets}

We next sought to identify the functional profiling of target genes of the 15 validated miRNAs. For that purpose, a Gene Ontology (GO) Enrichment Analysis was performed using Panther Classification System (http://pantherdb.org/). Only those target genes with strong evidence based on functional experiments, according to miRTarBase 8.0 (last accessed 09-22-2020), were considered. Interestingly, most of the enriched pathways were significantly associated with processes related to angiogenesis, regulation of endothelial cells or vasodilatation. Crucial pathways for cardiac muscle cell differentiation and proliferation were also present in the enrichment analysis. Other enriched target genes were related to immune response and inflammatory processes, particularly some controlling leukocyte adhesion to vascular endothelial cells, IL-6 mediated signalling, Th1 response, macrophage differentiation or MyD88 dependent Toll-Like Receptor signal pathways (Figure $2 b$ ).

In order to find relevant molecules associated with COVID-19 pneumonia, we measured soluble plasma proteins whose mRNAs were targeted by the identified miRNAs, according to miRTarBase 8.0. We used GO enrichment analysis and Venn diagram tool (http://bioinformatics.psb.ugent.be/webtools/Venn/) to select relevant proteins regulated by several of the validated miRNAs. Thereby, potential targets of our candidate miRNAs associated with angiogenesis pathways (epidermal growth factor receptor, EGFR and vascular endothelial growth factor A, VEGFA), leukocyte adhesion to endothelium (intercellular adhesion molecule 1, ICAM-1), chemokines or cytokines crucial for immune surveillance and lymphocyte proliferation (CXCL12 and IL-21 receptor, IL-21R) or genes involved in cell growth and proliferation (insulin like growth factor receptor 1 , IGF1R) were selected for quantification by ELISA. A previous set of cytokine quantification assays performed in a pilot screening of plasma soluble proteins with COVID-19 and CAP patients revealed differences in the plasma levels of IL-11, IL-17 and the tissue inhibitor of metalloproteinases 2 (TIMP-2) (Supplementary Fig. 4). Hence, these molecules were also quantified in the validation cohort. Finally, IL-10 was also included due to its described relevance in COVID-19 [22].

ELISA assays revealed higher levels of EGFR and IL-10 in COVID-19 vs. CAP patients (Figure 2c). Conversely, CXCL12 was downregulated in COVID-19 patients. Both EGFR and CXCL12 were targets of 5 and 2 validated miRNAs, respectively (Figure $2 \mathrm{~d}$ ). Likewise, we found differences in the levels of several molecules depending 
on COVID-19 severity. TIMP-2 and IL-17 were upregulated, while IL-21R was downregulated, in severe cases (Figure 2e). The chemokine CXCL12 was also increased in severe vs. mild COVID-19 (Figure 2e). Interestingly, 3 out of 8 subjects with higher levels of CXCL12 (above $15 \mathrm{pg} / \mathrm{ml}$ ) had bacterial superinfection or sepsis.

\section{Discussion}

Growing interest on miRNAs as potential biomarkers or as therapeutic drugs has raised over the last few years, especially in cancer, cardiovascular or neurodegenerative diseases. In this regard, miRNAs may have greater relevance in the future, since they provide us with a better understanding of the pathogenic mechanisms involved in different diseases. Although numerous publications concerning infection by SARSCoV-2 have been reported in the last year, very few studies addressed its relationship with miRNAs alterations. In silico analyses have focused on prediction of miRNAs targeting SARS-CoV-2 genome to find alternative therapies $[12,23]$. The present study describes a specific miRNA signature in the plasma of COVID-19 patients. A total of 15 miRNAs (7 upregulated and 8 downregulated in COVID-19), with common expression in human plasma and validated in an independent cohort appeared dysregulated in COVID-19 patients compared to CAP patients, revealing important differences in the pathophysiology of these two clinical entities despite their similarities in terms of respiratory symptoms. Due to these differences, we were able to develop a multivariate logistic regression model based on miRNAs that efficiently distinguishes patients with COVID-19 from patients with CAP.

Our study provides experimental evidence that confirms previous in silico bioinformatic analyses of possible miRNAs interacting with SARS-CoV-2 genome or playing a role in the host response to the virus. Particularly, dysregulation of miR-424-5p, miR-146a-5p, miR-130a-3p, miR-25-3p, miR-27b-3p and miR-425-5p was predicted in other studies, but it was not verified experimentally [24]. Among the miRNAs identified in our study, miR-335-5p was significantly downregulated in COVID-19 vs. CAP patients. This miRNA has been previously associated with suppression of inflammatory processes [25]. Its repression during SARS-CoV-2 infection may contribute to the widely described general proinflammatory status [26]. In line with these observations, we found low levels of plasma miR-146a-5p in COVID-19 vs. CAP patients. miR-146a regulates inflammation by targeting TNF receptor associated factor 6 (TRAF6), therefore reducing expression of NF-kB $[27,28]$. Moreover, decreased levels of miR-146a have been linked to higher risk of thrombotic events and neutrophil NETosis [29].

GO enrichment analysis revealed a potential involvement of vascular system biology in this pathology, specifically angiogenesis and response to endothelial damage. This is consistent with several articles that report atherosclerotic plaques, prothrombotic changes in endothelium, increased intussusceptive angiogenesis and a subsequent enhanced risk of thrombosis [30, 31]. EGFR is a protein involved in a great number of biological processes; some of them related to blood vessel growth, inflammation via NF-kB or profibrotic and atherosclerotic events. In this context, EGFR, a known target of miR-27b-3p, miR-146a-5p, miR16-5p, miR-335-5p and miR-30a-5p, is found at higher levels in patients with COVID-19, likely enhancing these events [32-34].

Intriguingly, we observed increased levels of the chemokine CXCL12 in CAP vs. COVID-19 patients. CXCL12, which is the CXCR4 ligand, is necessary for effective hematopoiesis, $T$ cell and memory B cell homing to the 
lymph nodes or monocyte recruitment. Inhibition of this axis is used by several viruses in order to increase their proliferation by reducing the number of circulating immune cells [35]. Whether low levels of this chemokine in COVID-19 patients could be triggered by SARS-CoV-2 is a possibility that merits further exploration. miR-146a-5p, miR-221-3p and their target CXCL12 (according to miRTarBase), were downregulated in COVID-19 patients. This observation suggests either non-canonical regulation by these miRNAs or the prevalence of other regulatory mechanisms modulating the expression and secretion of CXCL12. Moreover, CXCL12 was found at higher levels in the plasma of severe vs. mild COVID-19 patients. In our cohort, 3 severe COVID-19 patients, with higher levels of CXCL12, had sepsis or bacterial superinfection. Thus, we cannot rule out the relationship between higher levels of this chemokine and the presence of superinfection or sepsis in critically ill COVID-19 patients, since CXCL12 has a role in neutrophil recruitment from bone marrow [36].

IL-17 was found at higher levels in patients with severe COVID-19 disease, in agreement with a prior study indicating its crucial role in the pathogenesis of acute respiratory distress syndrome (ARDS) [37]. IL-17 induces recruitment of neutrophils to the lung, exacerbating proinflammatory cytokine release and leading to ARDS. Therefore, the IL-17 signalling pathway could be a potential target to treat the cytokine storm observed in the most severe COVID-19 cases [38]. Another well-studied cytokine in the pathogenesis of this disease is IL-10. Several published works show an increase of IL-10 in critically ill COVID-19 patients despite its general anti-inflammatory nature, even proposing this protein as a prognostic biomarker [39, 40], together with other well-known inflammatory cytokines such as IL-6. Here, we report an increased amount of IL-10 in COVID-19 vs. CAP patients. Conversely, no differences were observed between mild and severe disease. Finally, IL-21 is involved in antiviral defence by enhancing Th1 and IFN- $\gamma$ responses [41]. Likewise, its role for a correct B cell memory differentiation and germinal centre reaction leading to effective antibody response could be decisive for viral clearance [42]. Thus, low levels of its receptor, IL-21R, in severe COVID-19 could lead to an impaired IFN expression and facilitate the spread and replication of SARS-CoV-2. Receptor proteins like IL-21R or EGFR may be found in plasma by leakage from different cells and tissues. However, caution should be taken when interpreting these results, since soluble EGFR may act as a regulator of its signalling pathway, as previously described [43].

On the other hand, our data revealed clear differences in TIMP-2 levels between severe and mild cases of COVID-19. One of the functions of this inhibitor of matrix metalloproteinases (MMP) is to participate in the regulation of the renewal of the extracellular matrix. Besides this role, it was proposed as an inhibitor of angiogenesis by MMP-independent mechanisms [44]. Therefore, this protein may be participating in the response to direct endothelial damage caused by SARS-CoV-2.

Despite size sample limitation, this study conclusively proves the existence of a differential profile in circulating miRNAs between COVID-19 and CAP patients. Analysis of the identified miRNAs showed regulatory functions associated with angiogenesis and inflammation, indicating that endothelial damage and vascular compromise is definitively one of the main conditions driven by SARS-CoV-2. Here, we describe new miRNAs and soluble cytokines that may contribute to gain insight into COVID-19 pathogenic mechanisms and set the basis for the urgently needed design of novel therapeutic strategies. 


\section{Declarations}

\section{Ethics approval}

Transfer of samples from the Biobank and the study protocol was approved by the local Research Ethics Committee (register number 4070) and it was carried out following the ethical principles established in the Declaration of Helsinki.

\section{Consent for publication}

All recruited patients (or their representatives) were informed about the study and gave an oral informed consent as proposed by AEMPS due to COVID-19 emergency.

\section{Availability of data and material}

The data that support the findings of this study are available from the corresponding author upon reasonable request.

\section{Funding}

This study was funded by Spanish Ministry of Economy, Industry and Competitiveness (MINECO) and Instituto de Salud Carlos III (grant nos. RD16/0011/0012 and PI18/ 0371 to IGA, grant no. PI19/00549 to AA, and grant no. PDI-2020-120412RB-I00 to FSM) and co-funded by the European Regional Development Fund. The study was also funded by "La Caixa Banking Foundation" (grant no. HR17-00016 to FSM) and "Fondos Supera COVID19" by Banco de Santander and CRUE. The work of ERV has been funded by a Rio-Hortega grant from the Ministerio de Economía y Competitividad (grant no. CM19/00149 Instituto de Salud Carlos III) and co-funded by The European Regional Development Fund (ERDF) "A way to make Europe".

\section{Acknowledgments}

The authors would like to thank Dr. Miguel Vicente-Manzanares for manuscript revision and editing. They also acknowledge Dr. Mar Valés-Gómez, Dr. José Miguel Rodríguez-Frade and José M. Casasnovas for proving the SARS-CoV-2 recombinant $S$ and NP proteins.

\section{Author contributions}

Conception and design: PMF, PVT, MJF, SR, CLS, MLP, HF, FSM. Acquisition of data and samples: PMF, PVT, ERV, AV, ALS, JMGR, JA. Statistical analyses: PMF, PVT, MJF, SR, AS. Drafting the manuscript: PMF, PVT. Revising the manuscript: PMF, PVT, MJF, SR, MLP, ERV, AS, IGA, AA, CMC, JMGR, JA, HF, FSM, EMG. Obtaining financial support: HF, FSM.

\section{Competing interest statement}

The authors declare that they have no competing interests that have influenced the generation or interpretation of the data included here. 


\section{References}

1. Zhu N, Zhang D, Wang W et al (2020) A Novel Coronavirus from Patients with Pneumonia in China, 2019. N Engl J Med 382:727-733. https://doi.org/10.1056/nejmoa2001017

2. Nalbandian A, Sehgal K, Gupta A et al (2021) Post-acute COVID-19 syndrome. Nat Med 27:601-615. https://doi.org/10.1038/s41591-021-01283-z

3. Henry BM, De Oliveira MHS, Benoit S et al (2020) Hematologic, biochemical and immune biomarker abnormalities associated with severe illness and mortality in coronavirus disease 2019 (COVID-19): A meta-analysis. Clin Chem Lab Med 58:1021-1028. doi 10.1515/cclm-2020-0369

4. Rodríguez-Serrano DA, Roy-Vallejo E, Zurita Cruz ND et al (2021) Detection of SARS-CoV-2 RNA in serum is associated with increased mortality risk in hospitalized COVID-19 patients. Sci Rep 11:13134. https://doi.org/10.1038/s41598-021-92497-1

5. Sims JT, Krishnan V, Chang CY et al (2021) Characterization of the cytokine storm reflects hyperinflammatory endothelial dysfunction in COVID-19. J Allergy Clin Immunol 147:107-111. https://doi.org/10.1016/j.jaci.2020.08.031

6. Ambros V (2004) The functions of animal microRNAs. Nature 431:350-355. https://doi.org/10.1038/nature02871

7. Valadi H, Ekström K, Bossios A et al (2007) Exosome-mediated transfer of mRNAs and microRNAs is a novel mechanism of genetic exchange between cells. Nat Cell Biol 9:654-659. https://doi.org/10.1038/ncb1596

8. Lu LF, Boldin MP, Chaudhry A et al (2010) Function of miR-146a in Controlling Treg Cell-Mediated Regulation of Th1 Responses. Cell 142:914-929. https://doi.org/10.1016/j.cell.2010.08.012

9. Mittelbrunn M, Sánchez-Madrid F (2012) Intercellular communication: Diverse structures for exchange of genetic information. Nat Rev Mol Cell Biol 13:328-335. doi 10.1038/nrm3335

10. Trobaugh DW, Klimstra WB (2017) MicroRNA Regulation of RNA Virus Replication and Pathogenesis. Trends Mol Med 23:80-93. doi 10.1016/j.molmed.2016.11.003

11. Zheng B, Zhou J, Wang H (2020) Host microRNAs and exosomes that modulate influenza virus infection. Virus Res 279:197885. doi 10.1016/j.virusres.2020.197885

12. Fulzele S, Sahay B, Yusufu I et al (2020) COVID-19 virulence in aged patients might be impacted by the host cellular MicroRNAs abundance/profile. Aging Dis 11:509-522. https://doi.org/10.14336/AD.2020.0428

13. Demirci MDS, Adan A (2020) Computational analysis of microRNA-mediated interactions in SARS-CoV-2 infection. PeerJ 8:e9369. https://doi.org/10.7717/peerj.9369

14. Curbelo J, Bueno SL, Galván-Román JM et al (2017) Inflammation biomarkers in blood as mortality predictors in community-acquired pneumonia admitted patients: Importance of comparison with neutrophil count percentage or neutrophil-lymphocyte ratio. PLoS One 12:e0173947. https://doi.org/10.1371/journal.pone.0173947

15. Galván-Román JM, Lancho-Sánchez Á, Luquero-Bueno S et al (2020) Usefulness of circulating microRNAs miR-146a and miR-16-5p as prognostic biomarkers in community-acquired pneumonia. PLoS 
One 15:e0240926. https://doi.org/10.1371/journal.pone.0240926

16. Metlay JP, Waterer GW, Long AC et al (2019) Diagnosis and treatment of adults with community-acquired pneumonia. Am J Respir Crit Care Med 200:e45-e67. https://doi.org/10.1164/rccm.201908-1581ST

17. COVID-19 Treatment Guidelines Panel. Coronavirus Disease 2019 (COVID-19). Treatment Guidelines. National Institutes of Health. Available at https://www.covid19treatmentguidelines.nih.gov/. Accessed [06/2020]

18. Marabita F, De Candia P, Torri A et al (2016) Normalization of circulating microRNA expression data obtained by quantitative real-time RT-PCR. Brief Bioinform 17:204-212. https://doi.org/10.1093/bib/bbv056

19. D’haene B, Mestdagh P, Hellemans J, Vandesompele J (2012) miRNA expression profiling: From reference genes to global mean normalization. Methods Mol Biol 822:261-272. https://doi.org/10.1007/978-1-61779-427-8_18

20. Martínez-Fleta P, Alfranca A, González-Álvaro I et al (2020) SARS-CoV-2 Cysteine-like Protease Antibodies Can Be Detected in Serum and Saliva of COVID-19-Seropositive Individuals. J Immunol 205:31303140. https://doi.org/10.4049/jimmunol.2000842

21. Benjamini Y, Hochberg Y (1995) Controlling the False Discovery Rate: A Practical and Powerful Approach to Multiple Testing. J R Stat Soc Ser B 57:289-300. https://doi.org/10.1111/j.25176161.1995.tb02031.x

22. Song JW, Zhang C, Fan X et al (2020) Immunological and inflammatory profiles in mild and severe cases of COVID-19. Nat Commun 11:3410. https://doi.org/10.1038/s41467-020-17240-2

23. Alam T, Lipovich L (2021) miRCOVID-19: Potential Targets of Human miRNAs in SARS-CoV-2 for RNABased Drug Discovery. Non-coding RNA 7:18. https://doi.org/10.3390/ncrna7010018

24. Yousefi H, Poursheikhani A, Bahmanpour Z et al (2020) SARS-CoV infection crosstalk with human host cell noncoding-RNA machinery: An in-silico approach. Biomed Pharmacother 130:110548. https://doi.org/10.1016/j.biopha.2020.110548

25. Zhang Z, Chen L, Xu P et al (2020) Gene correlation network analysis to identify regulatory factors in sepsis. J Transl Med 18:381. https://doi.org/10.1186/s12967-020-02561-z

26. Blanco-Melo D, Nilsson-Payant BE, Liu WC et al (2020) Imbalanced Host Response to SARS-CoV-2 Drives Development of COVID-19. Cell 181:1036-1045.e9. https://doi.org/10.1016/j.cell.2020.04.026

27. Deng Y, Yan Y, Tan K, Sen et al (2017) MicroRNA-146a induction during influenza H3N2 virus infection targets and regulates TRAF6 levels in human nasal epithelial cells (hNECs). Exp Cell Res 352:184-192. https://doi.org/10.1016/j.yexcr.2017.01.011

28. Su YL, Wang X, Mann M et al (2020) Myeloid cell-targeted miR-146a mimic inhibits NF-kB-driven inflammation and leukemia progression in vivo. Blood 135:167-180. https://doi.org/10.1182/blood.2019002045

29. Arroyo AB, Fernández-Pérez MP, Del Monte A et al (2020) miR-146a is a pivotal regulator of neutrophil extracellular trap formation promoting thrombosis. Haematologica 106:1636-1646. https://doi.org/10.3324/haematol.2019.240226 
30. Ackermann M, Verleden SE, Kuehnel M et al (2020) Pulmonary Vascular Endothelialitis, Thrombosis, and Angiogenesis in Covid-19. N Engl J Med 383:120-128. https://doi.org/10.1056/nejmoa2015432

31. Poznyak AV, Bezsonov EE, Eid AH et al (2021) Ace2 is an adjacent element of atherosclerosis and covid19 pathogenesis. Int J Mol Sci 22:4691. doi 10.3390/ijms22094691

32. Wang L, Huang Z, Huang W et al (2017) Inhibition of epidermal growth factor receptor attenuates atherosclerosis via decreasing inflammation and oxidative stress. Sci Rep 8:45917. https://doi.org/10.1038/srep45917

33. Amin DN, Bielenberg DR, Lifshits E et al (2008) Targeting EGFR activity in blood vessels is sufficient to inhibit tumor growth and is accompanied by an increase in VEGFR-2 dependence in tumor endothelial cells. Microvasc Res 76:15-22. https://doi.org/10.1016/j.mvr.2008.01.002

34. Vagapova ER, Lebedev TD, Prassolov VS (2021) Viral fibrotic scoring and drug screen based on MAPK activity uncovers EGFR as a key regulator of COVID-19 fibrosis. Sci Rep 11:11234.

https://doi.org/10.1038/s41598-021-90701-w

35. Arnolds KL, Spencer JV (2014) CXCR4: A virus's best friend? Infect Genet Evol 25:146-156. doi 10.1016/j.meegid.2014.04.018

36. Delano MJ, Kelly-Scumpia KM, Thayer TC et al (2011) Neutrophil Mobilization from the Bone Marrow during Polymicrobial Sepsis Is Dependent on CXCL12 Signaling. J Immunol 187:911-918. https://doi.org/10.4049/jimmunol.1100588

37. Muir R, Osbourn M, Dubois AV et al (2016) Innate lymphoid cells are the predominant source of IL-17A during the early pathogenesis of acute respiratory distress syndrome. Am J Respir Crit Care Med 193:407-416. https://doi.org/10.1164/rccm.201410-17820C

38. Lin X, Fu B, Yin S et al (2021) ORF8 contributes to cytokine storm during SARS-CoV-2 infection by activating IL-17 pathway. iScience 24:102293. https://doi.org/10.1016/j.isci.2021.102293

39. Zhao Y, Qin L, Zhang P et al (2020) Longitudinal COVID-19 profiling associates IL-1RA and IL-10 with disease severity and RANTES with mild disease. JCI Insight 5:e139834. https://doi.org/10.1172/jci.insight.139834

40. Han H, Ma Q, Li C et al (2020) Profiling serum cytokines in COVID-19 patients reveals IL-6 and IL-10 are disease severity predictors. Emerg Microbes Infect 9:1123-1130. https://doi.org/10.1080/22221751.2020.1770129

41. Strengell M, Matikainen S, Sirén J et al (2003) IL-21 in Synergy with IL-15 or IL-18 Enhances IFN-y Production in Human NK and T Cells. J Immunol 170:5464-5469. https://doi.org/10.4049/jimmunol.170.11.5464

42. Rankin AL, MacLeod H, Keegan S et al (2011) IL-21 Receptor Is Critical for the Development of Memory B Cell Responses. J Immunol 186:667-674. https://doi.org/10.4049/jimmunol.0903207

43. Maramotti S, Paci M, Manzotti G et al (2016) Soluble epidermal growth factor receptors (sEGFRs) in cancer: Biological aspects and clinical relevance. Int J Mol Sci 17:593. doi 10.3390/ijms17040593

44. Seo DW, Li H, Guedez L et al (2003) TIMP-2 Mediated Inhibition of Angiogenesis: An MMP-Independent Mechanism. Cell 114:171-180. doi 10.1016/s0092-8674(03)00551-8 
a

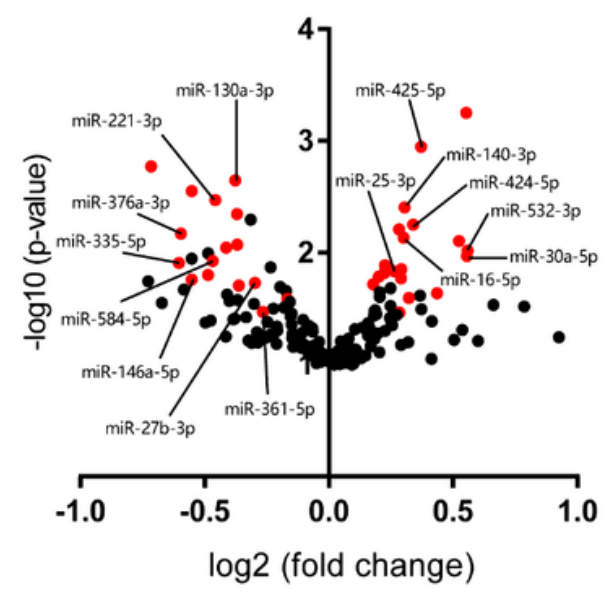

b

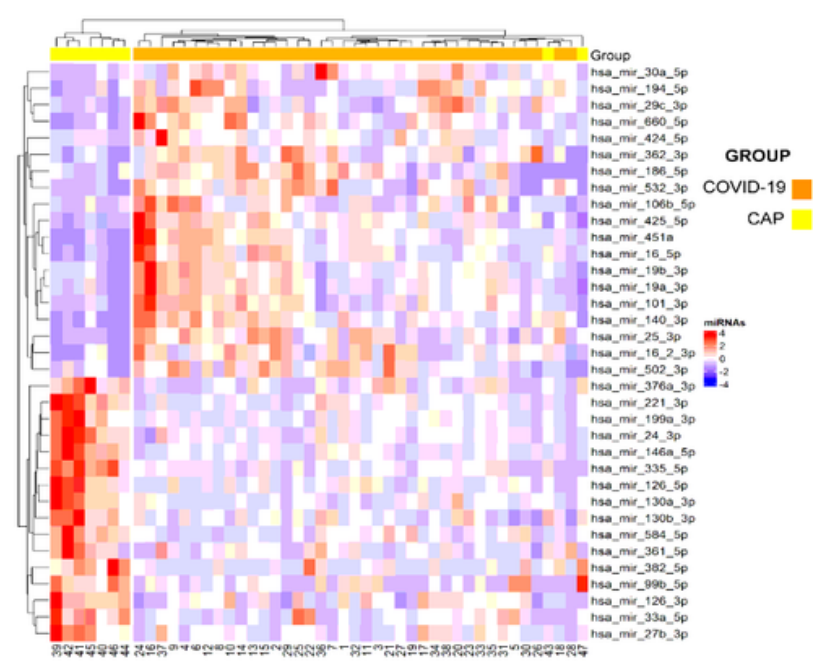

$\mathrm{c}$
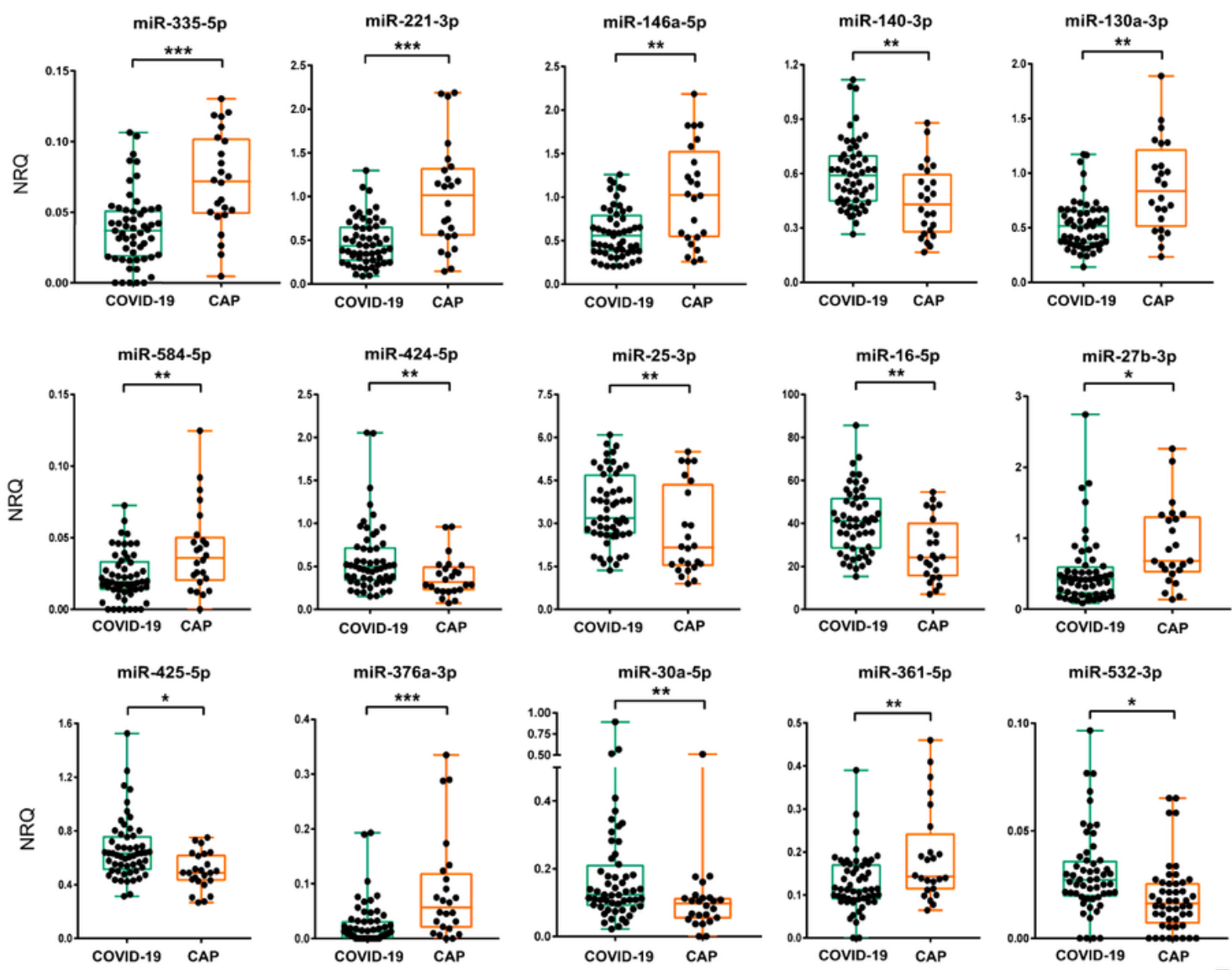

Figure 1

\section{Figure 1}

miRNA signature in COVID-19 patients. a) Volcano plot showing differential expression of 179 abundant miRNAs in human plasma between CAP and COVID-19 patients. Log2 of fold change of normalised relative quantities (NRQ) and statistical significance (- $\log 10$ of the p-value) from Mann-Whitney tests for each miRNA were assessed. In red, miRNAs with a corrected $p$ value $<0.05$ assessed by multivariate statistical 
analysis. Names of the 15 validated miRNAs are shown. b) Hierarchical clustering heatmap of 35 differentially expressed miRNAs in plasma of 9 CAP and 38 COVID-19 individuals. Red and blue colour indicate upregulated and downregulated expression of COVID-19, respectively, as compared to CAP. c) RTqPCR of 15 validated miRNAs performed in the validation cohort. Box and whisker plots of NRQ for each miRNA are shown. A group of 4 highly stable miRNAs were used as normalisers. Statistical significance was assessed by means of multivariate statistical tests. ${ }^{\star} p<0.05,{ }^{\star \star} \mathrm{p}<0.01,{ }^{\star \star \star} \mathrm{p}<0.001$.

a

\begin{tabular}{|c|c|c|c|c|}
\hline miRNA & OR & \multicolumn{2}{|c|}{$\mathrm{Cl} 95 \%$} & p-value \\
\hline Hemolysis & 3.5155 & 1.1353 & 18.062 & 0.1200 \\
\hline hsa-miR-106b-5p & 1000.01 & 8.8843 & $7.2608 \times 10^{5}$ & 0.0364 \\
\hline hsa-miR-221-3p & $2.5533 \times 10^{-5}$ & $8.2152 \times 10^{-9}$ & 0.0031 & 0.0043 \\
\hline hsa-miR-25-3p & 0.20365 & 0.042140 & 0.55820 & 0.0322 \\
\hline hsa-miR-30a-5p & $3.9557 \times 10^{7}$ & 793.34 & $8.7356 \times 10^{14}$ & 0.0313 \\
\hline
\end{tabular}

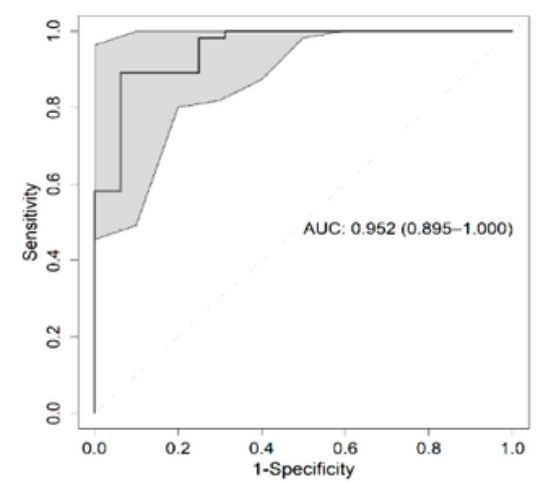

b

- $13.79 \%$ Cardiac cell metabolism and signalling

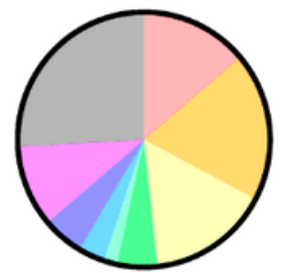

$18.97 \%$ Angiogenic processes

$15.52 \%$ Immune response and inflammation

= $5.17 \%$ Mitochondrial metabolism

- $1.72 \%$ Cellular senescence

= $3.45 \%$ Bone metabolism

= $5.17 \%$ Neuron signalling and metabolism

- $10.34 \%$ Generic cell signalling pathways

드 $25.86 \%$ Other pathways

c
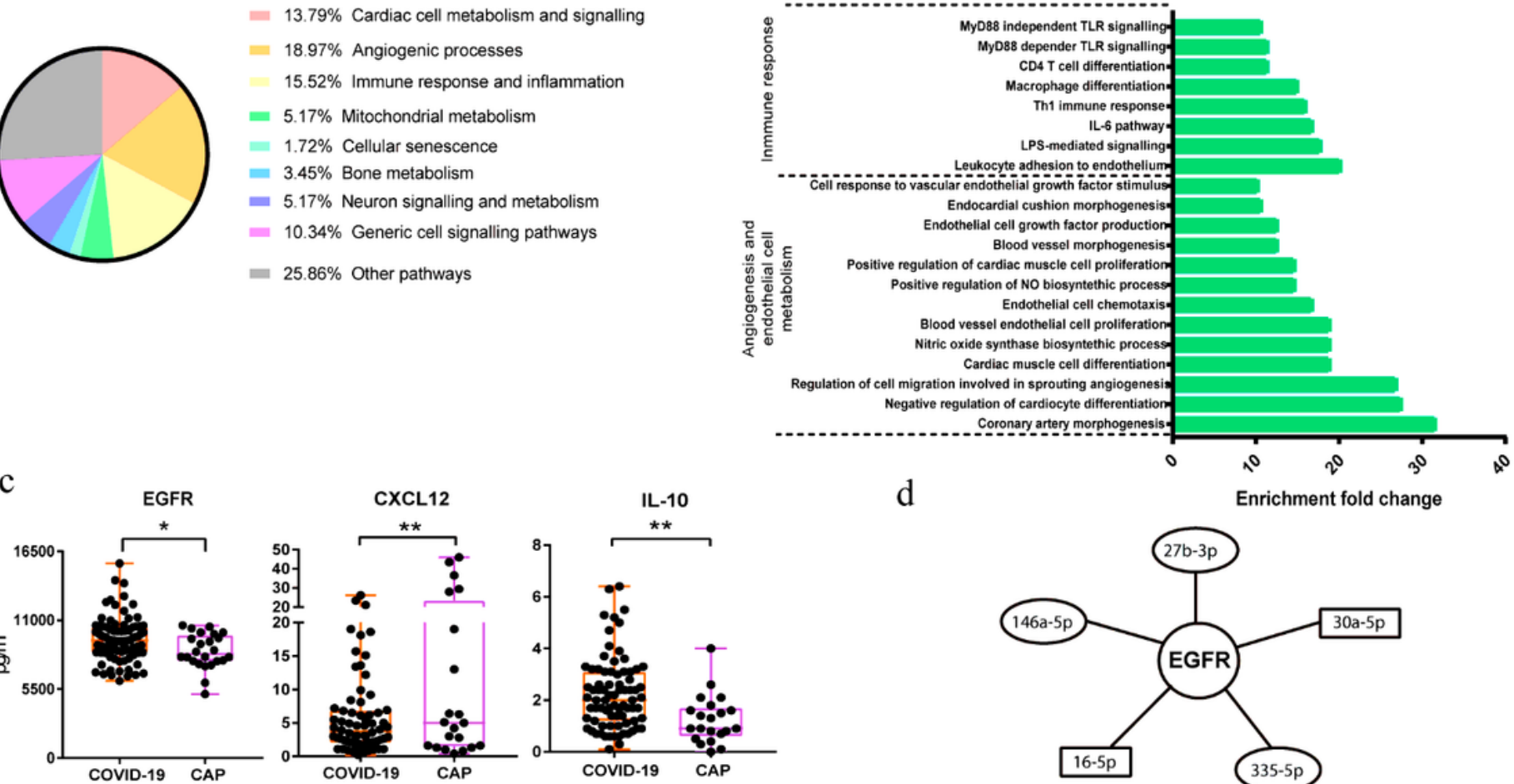

e
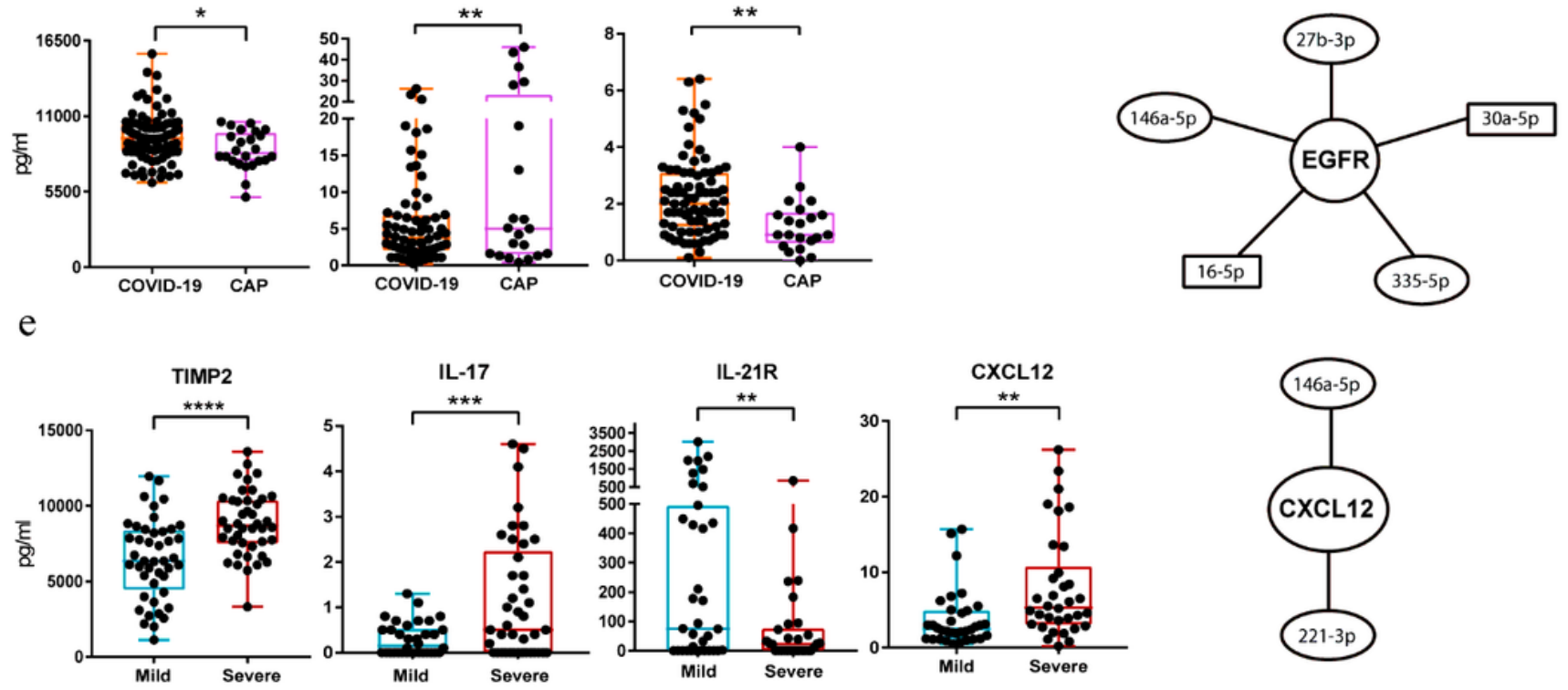

Figure 2

Figure 2 
Multivariate regression model for classification of COVID-19 pneumonia and CAP and differences in circulating proteins. a) Left: final logistic regression model for patient classification based on miRNAs. Odds ratio (OR), confidence interval 95\% $(95 \% \mathrm{Cl})$ and $\mathrm{p}$-value for each variable were calculated. Stepwise procedure, with both backward and forward search based on Akaike information criteria, to select the critical variables were employed. Right: ROC curve of the logistic regression model. Inside the graph, the AUC and its $95 \% \mathrm{Cl}$. b) Left: relative percentage of each biological process associated with the functional pathways with 10 fold enrichment or higher are shown. Pathway enrichment analysis was assessed using Panther Classification System. Right: enrichment of each individual functional pathway within the two most relevant biological processes. c) Measurement of soluble cytokines in plasma of 24 CAP and 85 COVID-19 individuals. ELISA assays with plasma diluted 1/2 were carried out. Box and whisker plots are shown, statistical significance was assessed by Mann-Whitney tests. ${ }^{*} p<0.05,{ }^{*} p<0.01$. d) miRNAs regulating EGFR and CXCL12 according to miRTarBase 8.0. Circles show miRNAs with lower expression in COVID-19 as compared to CAP. Rectangles show miRNAs with higher expression in COVID-19 as compared to CAP. e) Box and whisker plots of soluble cytokines in plasma of 43 mild and 42 severe COVID-19 patients. ELISA assays with plasma diluted 1/2 were carried out. Statistical significance was assessed by Mann-Whitney tests.

${ }^{* \star} p<0.01,{ }^{* *} p<0.001, * * \star * p<0.0001$.

\section{Supplementary Files}

This is a list of supplementary files associated with this preprint. Click to download.

- OnlineDataSupplementCMLS.pdf 